\title{
Rac1/ROCK-driven membrane dynamics promote natural killer cell cytotoxicity via granzyme-induced necroptosis
}

\author{
Yanting Zhu, Jun Xie and Jue Shi* (i)
}

\begin{abstract}
Background: Natural killer (NK) cells play an important role in cancer immunosurveillance and therapy. However, the target selectivity of NK cell activity is still poorly understood.

Results: Here, we used live-cell reporters to unravel differential epithelial cancer target killing by primary human NK cells. We found highly variable fractions of killing by distinct NK cell cytotoxic modes that were not determined by NK ligand expression. Rather, epithelial plasma membrane dynamics driven by ROCK-mediated blebs and/or Rac1mediated lamellipodia promoted necrotic mode in preference to the apoptotic mode of killing. Inhibition of granzyme B and key necroptosis regulators RIP1, RIP3, and MLKL significantly attenuated the necrotic killing, revealing a novel NK cell cytotoxic pathway by granzyme-induced necroptosis that conferred target selectivity.

Conclusions: Our results not only elucidate a new NK cell effector mechanism but also suggest that tissue microenvironment and oncogenic signaling pathways that promote membrane dynamics, e.g., Rac1 and Rho/ROCK, could be exploited to enhance proinflammatory NK cell killing.
\end{abstract}

Keywords: Natural killer cell, Cytotoxic dynamics, NK-cancer cell interaction, Cancer variability, Necroptosis, Membrane dynamics

\section{Background}

Natural killer (NK) cells detect and kill virus-infected cells and cancer cells and are considered a promising immunotherapeutic target $[1,2]$. Harnessing and stimulating the direct cytotoxic activity of NK cells has attracted growing interest in the recent development of cancer immunotherapy, as diverse tumor cell types showed susceptibility to NK cell killing and targeted therapy and immune checkpoint inhibitors may also act in part via triggering NK cell response [3-5]. Tumors are known to harbor large genetic and phenotypic heterogeneity that likely impact their differential interactions with NK cells. However, the mechanistic basis

\footnotetext{
* Correspondence: jshi@hkbu.edu.hk

Center for Quantitative Systems Biology, Department of Physics and Department of Biology, Hong Kong Baptist University, Kowloon, Hong Kong, China
}

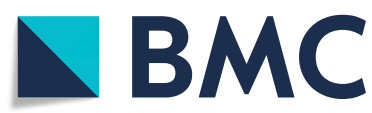

underlying the dynamic control over NK cells' selective killing of distinct target cell types is still poorly understood.

Conceptually, the variable sensitivity of cancer cells to killing by NK cells can be broken into two distinct processes, i.e., detection of abnormal cancer targets and selection of killing mechanism from the NK cell arsenal, which becomes important if the cancer target is resistant to one or more killing pathways. Of the two processes, target detection has been much more studied than the choice of killing mode. NK cells are known to detect abnormal cells by integrating signals from various inhibitory and activating receptors on their surfaces $[6,7]$. The major inhibitory receptors on NK cells are killer cell immunoglobulin-like receptors (KIRs), which interact with major histocompatibility complex I (MHCI) molecules of the target cells, leading to NK cell "self-

(c) The Author(s). 2021 Open Access This article is licensed under a Creative Commons Attribution 4.0 International License, which permits use, sharing, adaptation, distribution and reproduction in any medium or format, as long as you give appropriate credit to the original author(s) and the source, provide a link to the Creative Commons licence, and indicate if changes were made. The images or other third party material in this article are included in the article's Creative Commons licence, unless indicated otherwise in a credit line to the material. If material is not included in the article's Creative Commons licence and your intended use is not permitted by statutory regulation or exceeds the permitted use, you will need to obtain permission directly from the copyright holder. To view a copy of this licence, visit http://creativecommons.org/licenses/by/4.0/. The Creative Commons Public Domain Dedication waiver (http://creativecommons.org/publicdomain/zero/1.0/) applies to the data made available in this article, unless otherwise stated in a credit line to the data. 
tolerance" but activation when encountering "foreign" or transformed targets, such as tumor cells with low MHCI expression. Among the many different NK cell-activating receptors, NKG2D has been particularly implicated in NK cell-mediated immunity against tumors [8]. Ligands for NKG2D, such as MICA (MHC class I-related gene A), MICB (MHC class I-related gene $\mathrm{B}$ ), and ULBP (UL16-binding protein), are expressed in most human tumors [9], and inhibiting NKG2D function attenuated NK cell cytotoxicity against tumor targets [10].

The mechanisms underlying the choice of killing mode could depend on the sensitivity of the targets towards different modes and/or triggering of alternative cytotoxic pathways in the NK cells. NK cells are thought to kill targets by mechanisms, including lytic granule-mediated apoptosis, death ligand-mediated apoptosis, pyroptosis, and necrosis [11-15]. Lytic granule-mediated apoptosis is considered the principal cytotoxic pathway of NK cells. Upon the formation of NK-target cell immunological synapses, lytic granules release perforin and granzymes into the synapse. Granzymes then enter the target cell through the perforin channels, where they activate the apoptosis pathway via proteolytic activation of caspases [16]. In addition, NK cells express a variety of death ligands, such as Fas ligand (FasL) and TNF-related apoptosis-inducing ligand (TRAIL), which engage cognate death receptors expressed on the target cell surface, triggering death by receptor-mediated extrinsic apoptosis [17, 18]. Two recent studies identified pyroptosis as a new NK cell cytotoxic mechanism, in particular, activating proinflammatory cell death in tumors that express gasdermin B or gasdermin E $[13,14]$. Another highly proinflammatory NK cell killing mode is necrosis, which has been reported in a few studies and its mechanistic basis is poorly understood $[15,19]$. Here, we identified necroptosis as an NK cell cytotoxic effector mechanism for the first time. It may be a distinct cytotoxic mode, or equivalently underlying the necrotic killing that was previously reported. In light of the strong antitumor activity of NK cell-induced pyroptosis due to its proinflammatory consequences, the elucidation of necroptosis as a new proinflammatory NK cell killing mode is exciting, as this killing mode is not restricted by gasdermin expression and may be exploited for more diverse tumor subtypes.

To unravel the target selectivity of NK cell cytotoxicity, in this study, we characterized the rate-limiting kinetics and phenotypic heterogeneity of NK cell cytotoxic activity against a panel of normal and epithelial cancer targets using a live-cell Förster resonance energy transfer (FRET) reporter and quantitative single-cell imaging. Sensitivity of the epithelial target cells to overall NK cell killing was mainly conferred by the inhibitory KIRs-MHCI interaction, as expected. However, the choice of cytotoxic mechanism, e.g., necrosis vs. apoptosis, was determined by differential plasma membrane dynamics of the epithelial cell targets. In particular, a highly dynamic target membrane driven by Rac1 and/or Rho/ROCK activities, which are responsible for membrane protrusion and contractility, promoted granzyme B-induced actin depolymerization followed by target membrane leakage and formation of large blebs, ultimately leading to target cell death by the highly proinflammatory necroptosis. The elucidation of membrane dynamics as a novel cellular determinant for distinct NK cell killing mode is important because the cytotoxic mode determines the molecular pathways by which cancer cells might become resistant under selection, and also because it may influence downstream immunological consequences of killing. Moreover, previously characterized necroptosis is typically activated by death receptor in combination with caspase inhibition. Our finding that the necroptotic machinery can be alternatively activated by granzyme $\mathrm{B}$ expands the biology of necroptosis to the immunological context and has revealed an alternative signaling cascade to understand cytotoxic lymphocyte activity as well as necroptosis regulation.

\section{Results}

\section{Target cell-type dependence of NK cell cytotoxicity}

To investigate the mechanisms underlying the differential activity of NK cells on distinct targets, we chose a panel of human epithelial cell lines that exhibited variable sensitivity to primary NK cell killing, including one normal cell line, LO2 (immortalized normal hepatic cell line), and four cancer cell lines, i.e., HeLa (cervix cancer), SMMC-7721 (liver cancer), MCF7 (breast cancer), and U-2 OS (bone cancer). To visualize the dynamic response to NK cells, we engineered each epithelial cell line with a previously established fluorescent FRET reporter specific to granzyme B activity (GrzmB-FRET) [20] as well as a mitochondria reporter of apoptosis (IMS-RP) [21-23]. The GrzmB-FRET reporter consists of a cyan (CFP, donor) and yellow (YFP, receptor) fluorescent protein linked by a peptide substrate specific to granzyme B, i.e., VGPDFGR. Upon lytic granule transfer and release of granzyme B into the target cell, granzyme $B$ cleaves the peptide linker of the FRET reporter, and energy transfer from CFP to YFP is thus lost, resulting in a decrease of YFP fluorescence and increase of CFP fluorescence. All NK-target cell co-culture assays were conducted with human primary NK cells purified from healthy blood donors and pre-activated by IL-2 for 3 days, at an NK-to-target cell ratio of 3:1. Target cell death was scored morphologically by blebbing followed by cell lysis, and kinetics of target cell death from NK cell addition to morphological cell death was plotted as 
cumulative survival curves. As shown in Fig. 1a, the non-cancer cell line, LO2, was the most resistant to NK cell killing, consistent with NK cell's function in eliminating abnormal and cancerous cells. Among the cancer cell lines, SMMC-7721 and MCF7 were the most sensitive, with MCF7 exhibiting the fastest kinetics to cell death.

In addition to variable sensitivity to overall NK cell killing, we also observed a striking difference in the cytotoxic modes used to kill the different target cell lines. The majority of cell death events (about 60\%) seen in SMMC-7721 were preceded by a loss of the granzyme-B FRET (i.e., an increase of CFP (donor) signal and decrease of YFP (acceptor) signal) followed by mitochondrial outer membrane permeabilization (MOMP), suggesting that NK cells killed SMMC-7721 mainly by the lytic granule and granzyme B-mediated cytotoxic pathway and intrinsic apoptosis (Fig. 1b). In contrast,
NK cell cytotoxicity towards U-2 OS cells mostly did not associate with a change in the FRET signal of granzyme B. We have previously characterized this mode of target cell death to be mediated by the death ligand, e.g., FasL, and subsequent extrinsic apoptosis [22]. Interestingly, we found necrosis, a much less explored NK cell cytotoxic mechanism, was the primary cytotoxic mode that triggered cell death in MCF7 cells. As shown in the bottom panel of Fig. 1b, MCF7 cell death was not preceded by MOMP, indicating it was not classic apoptosis. Moreover, the signal from the granzyme B-FRET reporter was abruptly lost upon extensive MCF7 cell blebbing, pointing to a large-scale leakage of the intracellular content likely due to membrane ruptures. Such dynamic features were consistent with necrotic cell death. The variable extent of target cell death induced by NK cells was confirmed by flow cytometry analysis of Annexin V staining that measured total cell death (Additional file 1: Figure

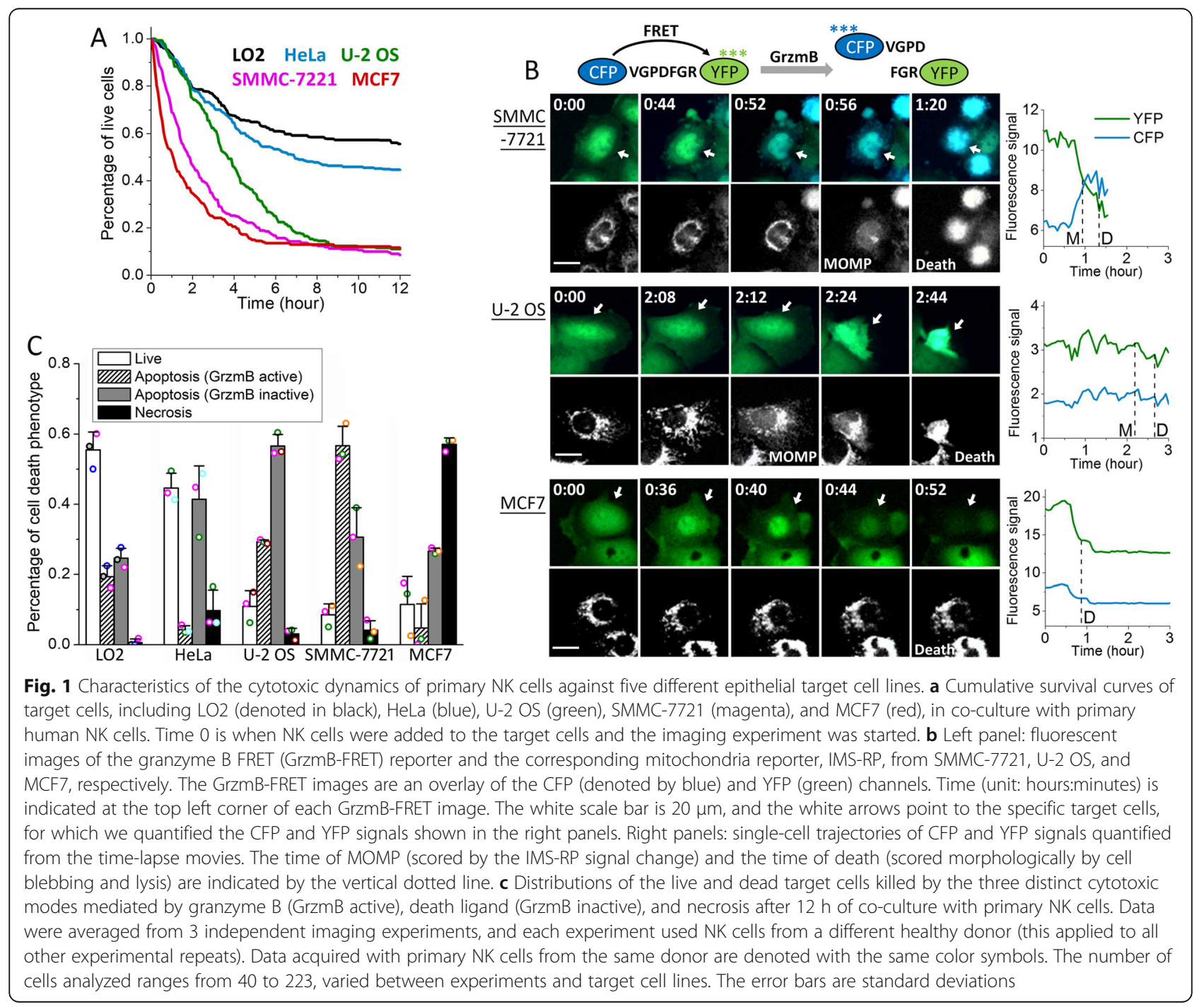


S1). However, such an ensemble method cannot distinguish the three distinct NK cell killing modes as revealed by our live-cell imaging assays.

Figure 1c summarized the percentage of live target cells and the cell death population via the three distinct cytotoxic modes of primary NK cells exhibited by the five epithelial target cell lines after $12 \mathrm{~h}$ of NK-target cell co-culture. The two most sensitive target cell lines, SMMC-7721 and MCF7, which showed rapid kinetics of cell death induction, were killed mainly (around 60\%) through granzyme B activity or necrosis, while cytotoxicity mediated by death ligand was more dominant in the less sensitive target cell types, U-2 OS, HeLa, and LO2. All target cell lines showed substantial cell death triggered by death ligands of NK cells, ranging from 25 to $57 \%$ of the total target cell population, suggesting the death receptor pathway is widespread. In contrast, the extent of cell death activated by the granzyme B and necrosis pathways varied more significantly, ranging from 4 to $56 \%$ for the lytic granule mode and 1 to $57 \%$ for the necrotic mode. Such large variability in the sensitivity to NK cell killing via granzyme $B$ and necrosis indicated that activation of these two cytotoxic modes may depend on epithelial features that are more cell type specific. All the cell death data analyzed in our study were averaged from primary NK cells from at least 3 different healthy blood donors. Variability between NK cells from the different donors could be estimated by the standard deviations shown as error bars in the data plots. Such interdonor variability is much smaller than the phenotypic difference that we characterized in terms of the variable sensitivity of different epithelial cell targets both to overall NK cell killing and to the distinct NK cell cytotoxic modes.

\section{Extent of cytotoxicity, but not cytotoxic mode, correlated with $\mathrm{MHCl}$ expression}

To investigate the potential molecular determinants underlying the observed variable sensitivity of epithelial cell lines to overall NK cell killing as well as to the distinct NK cell cytotoxic modes, we first profiled the expressions of NK cell-interacting surface molecules known to be involved in cancer-associated NK cell cytotoxicity for the selected target cell panel (Fig. 2a). These molecules include the human MHCI molecules (HLA-A, B,C and HLA-E), NKG2D ligands (MICA, MICB, and ULBP-2,5,6), and DNAM-1 ligands (PVR/CD155 and Nectin-2/CD112) [24]. We also measured the expression of the death receptor Fas and an integrin ligand key for NK cell conjugation, ICAM-1 (CD54), in our analysis by flow cytometry (Fig. 2b). The quantified FACS results showed highly variable expressions of all surface molecules that we profiled between the five target cell lines. The sensitivity of target cell lines to overall NK cell killing correlated relatively well with the expression levels of the inhibitory molecules, HLA-A,B,C and HLA$E$, and to a much lesser extent with MICB, but did not correlate with the expression of the other NKG2D activating ligands, MICA and ULBPs, or the activating ligands for DNAM-1. Surprisingly, the expression level of Fas did not correlate with the differential sensitivity to the death ligand-mediated NK cell cytotoxicity. Our data thus indicated that the inhibitory strength of KIRs-HLAA,B,C and NKG2A-HLA-E may exert the primary control over target cell recognition and overall NK cell cytotoxicity against a particular epithelial target, while control by the activating ligands is less universal and more context-dependent. However, our data did not show any significant correlative feature that was specific to the three distinct NK cell cytotoxic modes. The only differential ligand expression that may potentially render specificity to necrotic killing is MICB, as the MICB expression level in MCF7, the cell line that was particularly prone to necrotic death, was 5-10-folds higher than the other four cell lines. But we found RNAi knockdown of MICB in MCF7 cells did not attenuate necrotic death induced by NK cells (Additional file 1: Figure S2), indicating MICB expression was not the molecular determinant for the necrotic killing.

As we did not observe a correlation between the expressions of well-known NK cell-interacting surface ligands with the variable sensitivity to different NK cell cytotoxic modes, we went on to investigate the involvement of key inhibitory and activating receptors on NK cell surface, as the target specificity could be conferred by surface ligands beyond the profiling panel that we selected. The NK cell receptors that we examined included two types of inhibitory receptors, KIRs and NKG2A (CD94), and three activating receptors important for cancer target recognition, including NKG2D (CD314), DNAM-1 (CD226), and NKp46 (CD335) [11, 21]. Specifically, we used neutralizing antibodies to block individual receptors or a combination of the receptors and then compared the cytotoxic response via the three cytotoxic modes with those under the control condition (i.e., no receptor blocking). We used a broad-spectrum neutralizing antibody against HLA-A, HLA-B, and HLA-C to block the interaction of all KIRs and their MHCI binding partners as a whole, instead of examining the individual KIR, to simplify the analysis.

Figure 2c, d shows the receptor neutralizing results for HeLa cells in co-culture with primary NK cells. As expected, blocking the inhibitory NK-HeLa cell interaction via KIRs-HLA-A,B,C accelerated and enhanced HeLa cell killing, resulting in a degree of cell death similar to that observed in the sensitive target cell lines, such as SMMC-7721 (Fig. 2c). In contrast, blocking the other inhibitory receptor, NKG2A, did not alter cell death 


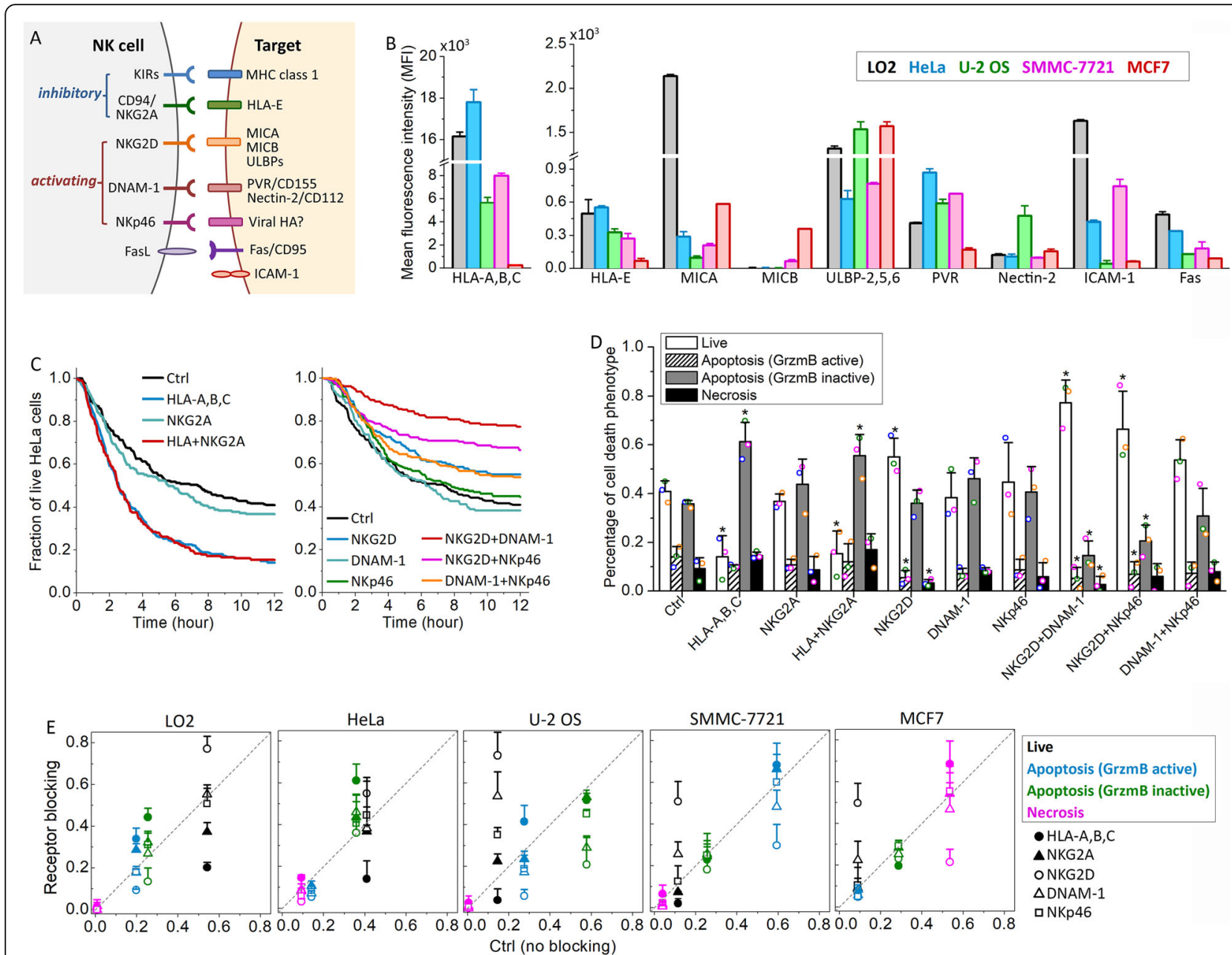

Fig. 2 Differential activation of the NK cell inhibitory and activating receptor signaling machinery upon interaction with the five epithelial target cell lines. a Diagram of the major NK cell receptors and their cognate ligands on the target cell. $\mathbf{b}$ Comparison of the surface protein expression in the five target cell lines based on the average florescent signal from the flow cytometry analysis. Cell lines were color-coded as indicated. Data were averaged from 3 independent flow cytometry analyses (> $1 \times 10^{4}$ cells in each analysis). The error bars are standard deviations. c Cumulative survival curves of HeLa cells in co-culture with primary NK cells in the presence of single or double blockage of the inhibitory receptor (left panel) or activating receptor (right panel). d Distributions of the live and dead HeLa cells killed by the three distinct cytotoxic modes after $12 \mathrm{~h}$ of co-culture with NK cells under the indicated treatment conditions. Data acquired with NK cells from the same donors are denoted with the same color symbols. P value was obtained by Student's $t$ test comparing the treatment condition with control. ${ }^{*} P<0.001$. e Percentage of live cells and cells that died via the three distinct cytotoxic modes under the different receptor-blocking conditions in comparison with those under the control condition. Data are color-coded as indicated. The different treatment conditions are denoted with the indicated symbols. For both $\mathbf{d}$ and $\mathbf{e}$, data plotted were averaged from 3 independent imaging experiments and the error bars are standard deviations

significantly. Neutralizing the activating receptors exerted a less prominent effect in attenuating the cell death response of $\mathrm{HeLa}$, possibly because $\mathrm{HeLa}$ cells under the control condition were already relatively resistant to primary NK cell killing. Inhibition of NKG2D activity exhibited a stronger effect in attenuating cell death than neutralizing DNAM-1 or NKp46 (Fig. 2c). Double blocking of NKG2D plus DNAM-1 or NKp46 further rescued HeLa cell death, confirming that the NK cell cytotoxicity is regulated by collective, rather than individual, signaling receptors.
We next investigated how blocking individual receptors altered induction of the three cytotoxic modes, expecting individual receptors to selectively regulate particular modes. However, this was not the result. Although blockage of the inhibitory KIRs-HLA-A,B,C mainly enhanced death ligand-mediated HeLa cell death, the fraction of NK cell killing mediated by granzyme B, death ligand, and necrosis all decreased in parallel upon neutralizing the activating receptor NKG2D, either alone or in combination with DNAM-1 or NKp46 (except for the apoptotic population via death ligand under NKG2D 
inhibition alone) (Fig. 2d). Therefore, in HeLa, receptor modulation appears to largely tune the overall cytotoxic activity of NK cells and/or receptivity of targets, but not the specific death pathway.

Figure $2 \mathrm{e}$ summarized the receptor neutralization results for all three pathways across the five epithelial target cell lines (detailed data can be found in Additional file 1: Figure S3). Here, we plotted the ratio of perturbation versus control. Intuitively, data points along the diagonal indicated no change relative to the control condition, and the further away the data points were from the diagonal, the larger the effect of the respective receptor inhibition in diverting the cell death response into, or away from, one of the three cytotoxic modes. Similar to HeLa cells, neutralization of the inhibitory KIRs-HLA-A,B,C interaction exerted a strong effect in enhancing the cell death response of the other four target cell lines, and loss of NKG2D activity exerted the strongest effect in attenuating target cell death. However, as of HeLa cells, the three cytotoxic modes were altered approximately in parallel, again showing that receptor modulation tuned the overall activity of NK cells, but not the activity of one specific death pathway. We also noted that except for MCF7, no other cell line showed significant cell death via necrosis under all receptor perturbation conditions, suggesting that MCF7 cells may have unique cellular features that promote the induction of necrotic killing, which we further investigated below.

It is possible that the receptor neutralization treatment may trigger another type of cell death, i.e., NK cellmediated antibody-dependent cell-mediated cytotoxicity (ADCC). To examine the involvement of ADCC, we blocked CD16, the key Fcy receptor on NK cells that mediates ADCC, and then co-cultured the CD16-neutralized NK cells with HeLa cells treated with HLA-A,B,C, neutralizing antibody, as HLA-A,B,C blocking showed the most significant effect in sensitizing HeLa cells to NK cell killing. We found blocking CD16 did not substantially attenuate NK cell killing under the HLA-A,B,C neutralizing antibody treatment, suggesting that ADCC did not contribute significantly to the NK cell killing that we observed (Additional file 1: Figure S4).

\section{Membrane dynamics modulate target sensitivity to NK cell cytotoxic modes}

Since receptor expression level did not predict which death pathway is preferred, and receptor inhibition did not modulate the fraction of death caused by a particular pathway, we sought other phenotypic properties that might predict and modulate individual NK cell cytotoxic pathways. We focused on MCF7 and SMMC-7721 because they had the most distinctive preferences, i.e., MCF7 cells were uniquely sensitive to necrosis, while
SMMC-7721 cells were sensitive to granzyme Bmediated intrinsic apoptosis. A distinguishing feature of these cell lines that we observed in our movies was the difference in plasma membrane dynamics. MCF7 cells exhibited extensive dynamic protrusions driven by membrane blebs and lamellipodia (Fig. 3a). In contrast, SMMC-7721 cells exhibited only moderate membrane dynamics and the membrane of U-2 OS cells was largely quiet (Fig. 3a). This observation led us to examine whether differential membrane dynamics of the epithelial cell targets contributed to the activation of alternative NK cell cytotoxic modes.

To attenuate the formation of dynamic membrane protrusions, we used either NSC23766, an inhibitor of the small GTPase Rac1, to inhibit actin polymerizationdriven lamellipodia, or Y27632, an inhibitor of the Rho Kinase (ROCK), to inhibit actomyosin contractility that promotes membrane blebs [25-27]. NSC23766 significantly reduced membrane protrusions in both MCF7 and SMMC-7721 cells, as evidenced by the kymograph of cell edge, which showed a relatively smooth edge under NSC23766 as compared to a jagged edge in the control cells (Fig. 3a). The effect of Y27632, however, diverged in MCF7 and SMMC-7721. MCF7 cells treated with Y27632 showed reduced membrane blebs but longer lamellipodia, a phenotype previously characterized as due to compensatory effects of membrane blebs and lamellipodia [25]. SMMC-7721 treated with Y27632 still showed evident membrane ruffling, indicating bleb did not contribute significantly to drive the membrane dynamics of SMMC-7721. Attenuation of membrane dynamics by both NSC23766 and Y27632 changed the primary cell death mode of MCF7 cells from necrosis to death ligand-mediated apoptosis, with NSC23766 exhibiting a slightly stronger effect (Fig. 3b). NSC23766 treatment of SMMC-7721 also resulted in a switch of NK cell killing from granzyme B-mediated apoptosis to death ligand-mediated apoptosis, while Y27632 did not show a significant effect on altering the NK cell cytotoxic mode (Fig. 3c). Moreover, we did not observe a distinctive change in the membrane distribution of NK ligand (e.g., HLA-A,B,C) on MCF7 cells or the NK receptor (e.g., KIR2D) on NK cell under either NSC23766 or Y27632 treatment, indicating that these two inhibitors did not alter NK cell killing modes by changing the receptor-ligand distribution in the plasma membrane (Additional file 1: Figure S5). Overall, our data suggested that inhibiting plasma membrane dynamics of epithelial targets promoted NK cell killing via death ligandmediated apoptosis, while damping the other two cytotoxic pathways.

Next, we examined whether increasing the membrane dynamics, e.g., in SMMC-7721, would alter NK cell killing from granzyme B-mediated apoptosis to the necrosis 

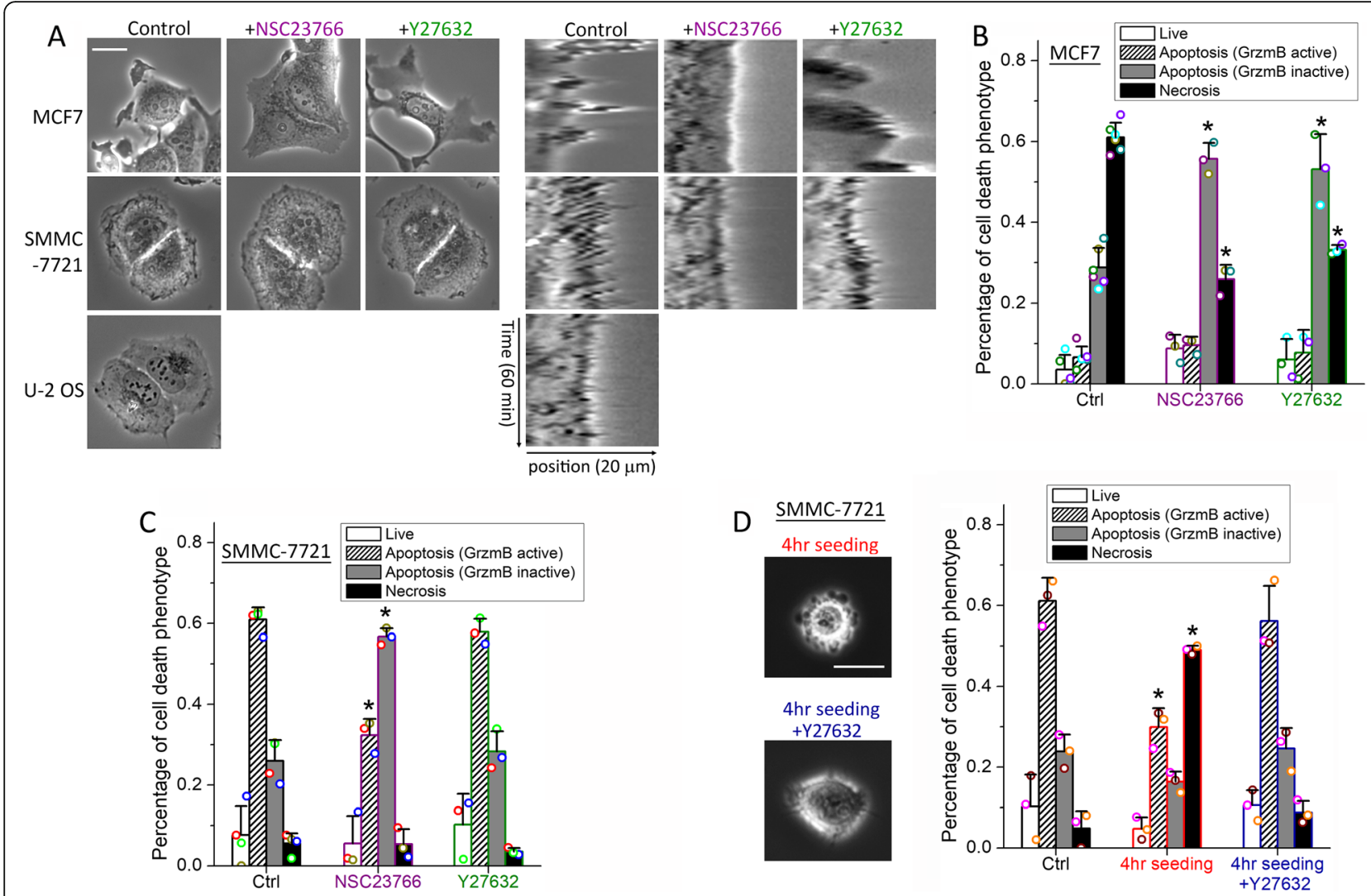

Fig. 3 Membrane dynamics of MCF7 and SMMC-7721 cells modulate NK cell cytotoxic modes. a Left panel: phase-contrast images of MCF7, SMMC-7721, and U-2 OS cells under control or treatment with NSC23766 (Rac1 inhibitor) or Y27632 (ROCK inhibitor). The white scale bar is 20 $\mu \mathrm{m}$. Right-panel: kymographs of a cell edge of MCF7, SMMC-7721, and U-2 OS cells under the indicated treatment conditions. The horizontal axis of the kymographs is the line position across the cell edges, spanning $20 \mu \mathrm{m}$, and the vertical axis is the time evolution from 0 to 60 min. $\mathbf{b}$, $\mathbf{c}$ Distributions of the cytotoxic response phenotypes of target cells under control or the indicated inhibitor treatment for $\mathbf{b}$ MCF7 cells or c SMMC7721 cells in co-culture with primary NK cells for $12 \mathrm{~h}$. $\mathbf{d}$ Phase-contrast images and the response phenotype distribution of SMMC-7721 cells that adhered to the cell culture plate for $4 \mathrm{~h}$ (with or without Y27632) and in co-culture with primary NK cells for $12 \mathrm{~h}$ in comparison with control cells that adhered for $24 \mathrm{~h}$. Data plotted in a-d were averaged from 3 independent imaging experiments, and the number of cells analyzed for each condition/cell line/experiment ranges from 44 to 149. The error bars are standard deviations. Data acquired with NK cells from the same donors are denoted with the same color symbols in each sub-figure. P value was obtained by Student's t test comparing the treatment condition with control. * $P<0.001$

mode, similar to MCF7. We noted that SMMC-7721 cells showed extensive membrane blebs when they started to adhere to the cell culture plate surface (Fig. 3d, left panel). And these blebs disappeared upon complete adherence (Fig. 3a, control). We therefore co-cultured primary NK cells with SMMC-7721 that were trypsinized and seeded onto the culture plate for only $4 \mathrm{~h}$ to investigate the effect of enhanced membrane dynamics, in this case, driven by blebs, on NK cell killing mode. Compared to control SMMC-7721 cells that had adhered to the culture plate for $24 \mathrm{~h}$, which were mainly $(\sim 60 \%)$ killed by granzyme B-mediated apoptosis, the majority $(\sim 50 \%)$ of the short-adherence cells were killed via the necrotic mode (Fig. 3d, right panel). And the addition of the bleb inhibitor, Y27632, abrogated the increase in necrotic killing of the short-adherence cells (Fig. 3d). As U-2 OS cells adhered to the culture plate surface very quickly (in about 3-5 h) and did not exhibit prolonged blebs, we were unable to use this experimental strategy to study the effect of enhanced membrane dynamics on promoting granzyme B-mediated apoptosis over the death ligand-mediated mode. Nonetheless, overall, our results suggested that the extent of membrane dynamics exerted a key control over the sensitivity of different epithelial targets to the three distinct NK cell cytotoxic modes.

\section{Pro-necrotic membrane dynamics involve actin depolymerization}

To determine how a highly dynamic target membrane promotes pro-necrotic killing, we stained the lytic granules of NK cells with an acidic organelle marker, LysoBrite, and investigated the collective dynamics of NK and target cells upon formation of the immunological 
synapse. NK cells typically showed polarized morphology with the acidic lytic granules stored in the tail end, as they moved in the co-culture environment. Such localization of lytic granules likely prevents undesirable leakage of lytic granules during the constant transient contacts between NK and target cells. When NK cells recognized an abnormal MCF7 cell target, a sustained conjugation called immunological synapse (IS) was formed and subsequently triggered the reorientation of lytic granule from the tail to the IS and then lytic granule transfer (Fig. 4a, left panel; Additional file 2: Movie S1). We found localization and transfer of lytic granule at IS were accompanied by large membrane blebs of MCF7 cell, which, within 4-8 min, ruptured the MCF7 cell membrane and caused necrotic death. We also noted the large membrane blebs tended to form at sites previously showing large lamellipodia (Fig. 4a, frames \#1 and \#2), suggesting possible conversion of lamellipodia to bleb. SMMC-7721, in comparison, exhibited significantly less membrane bleb upon the localization and transfer of lytic granules at the IS that was followed by the gradual accumulation of granzyme B activity as indicated by the loss of GrzmB-FRET signal, and induced apoptotic death in 30-40 min (Fig. 4a, right panel; Additional file 3: Movie S2). Attenuating MCF7 membrane dynamics by inhibiting Rac1 or ROCK activity abrogated the induction of large membrane bleb at the IS and subsequent necrotic killing (Fig. 4b). And increasing the
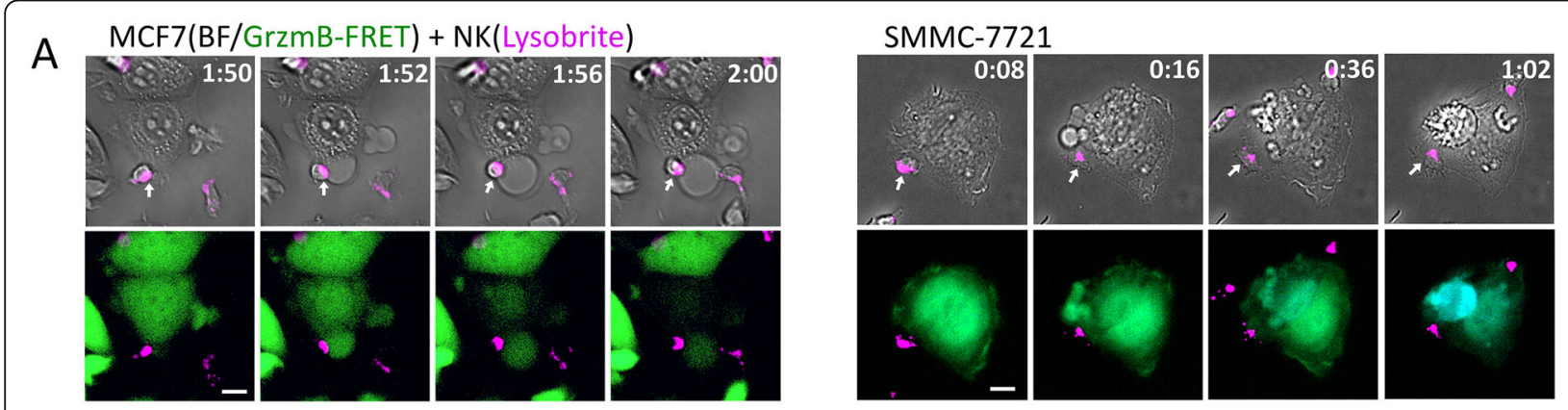

B $\quad$ MCF7 + NSC23766
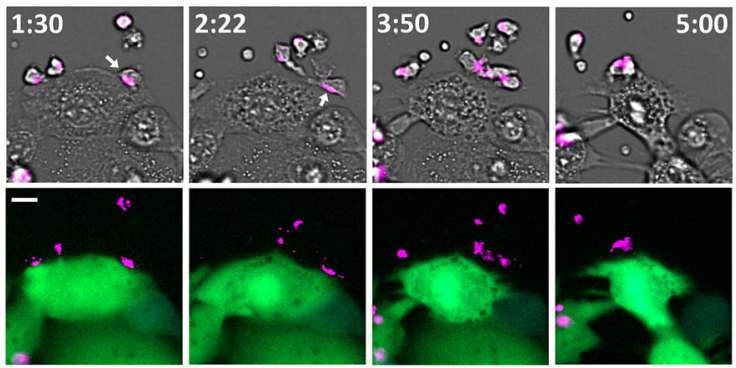

SMMC-7721 (4hr adherence)

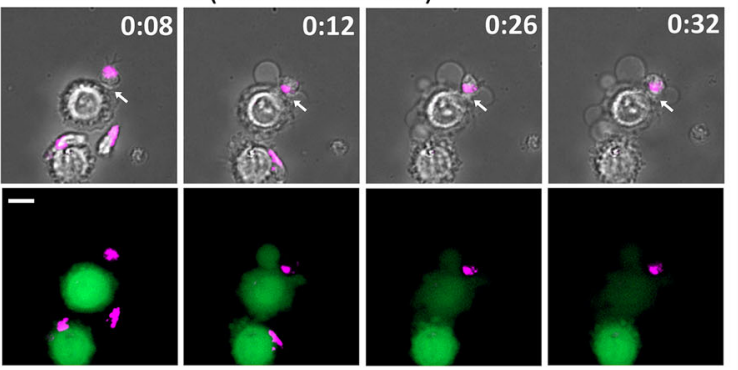

C MCF7(F-actin/PI) + NK(Lysobrite)

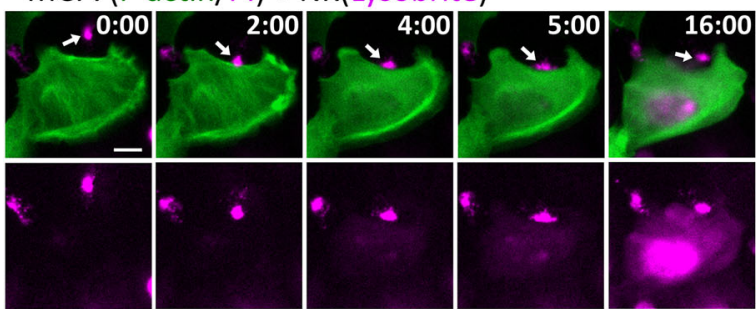

D MCF7(F-actin) + NK(Lysobrite)

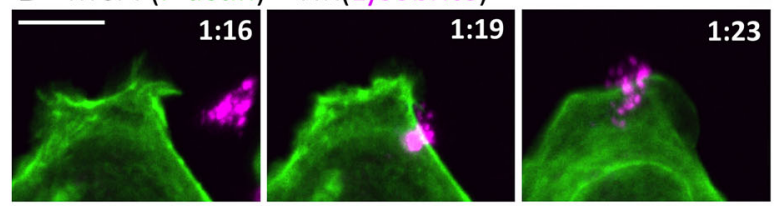

Fig. 4 Necrotic killing of NK cells are associated with actin depolymerization and membrane leakage. a Representative still images obtained from live-cell imaging of MCF7 (left panel) and SMMC-7721 (right panel) in co-culture with primary NK cells. The upper panel shows the overlay images of bright-field and the lytic granule marker, LysoBrite (denoted in magenta). The lower panel shows the overlay images of the GrzmBFRET reporter and LysoBrite. Time is indicated in the unit of hours:minutes at the top of the images. The specific interacting NK cell is indicated by the white arrows. b Still images of MCF7 cells treated with NSC23766 (Rac1 inhibitor, left panel) and SMMC-7721 cells upon 4-h adherence (right panel) in co-culture with primary NK cells stained with LysoBrite. c Dynamics of F-actin (denoted in green) and membrane leakage probed by PI (magenta, detected by the same fluorescence channel as LysoBrite) infiltration into the MCF7 cells triggered by NK cells. Time is indicated in the unit of minutes:seconds. The imaging frame rate for this time-lapse experiment is $30 \mathrm{~s}$ per frame. $\mathbf{d}$ Confocal live-cell imaging of F-actin (denoted in green) dynamics upon MCF7-NK cell interaction. NK cell was labeled with LysoBrite (magenta). Time is indicated in the unit of hours:minutes. The white scale bar in $\mathbf{a}-\mathbf{d}$ is $10 \mu \mathrm{m}$ 
membrane dynamics of SMMC-7721 by short adherence induced a large bleb at the IS and led to necrotic death, similar to the dynamic phenotype that we observed for MCF7 cells (Fig. 4b, right panel).

As the large membrane bleb was a key feature preceding necrotic killing, we further investigated its mechanistic origin. The observation of possible conversion of lamellipodia to large bleb led us to examine the dynamics of cytoskeleton, in particular F-actin, by imaging a transiently expressed Utrophin-GFP reporter in the MCF7 cells [28]. We also added propidium iodide (PI) in the live-cell imaging assay to determine the onset of membrane leakage in the necrotic killing process. As shown in Fig. 4c, lamellipodia immediately stalled and retracted upon IS formation and the actin cytoskeleton largely depolymerized within $2 \mathrm{~min}$. Membrane leakage indicated by PI diffusion into the MCF7 cell was generally observed 1-1.5 min subsequent to the change in actin dynamics (the PI fluorescent signal was detected in the same channel as LysoBrite). Confocal imaging of Factin and LysoBrite confirmed the change in actin dynamics and cytoskeleton upon lytic granule localization to the IS at higher spatial resolution (Fig. 4d; Additional file 4: Movie S3). Distinct from the MCF7 cells, IS formation in SMMC-7721 led to the alignment of F-actin into long fibers, likely due to increased contractility known in apoptotic cells (Additional file 5: Movie S4). Together our data suggested that lytic granules of NK cells induced actin deploymerization and loss of lamellipodia that initiated bleb formation, especially at the highly dynamic membrane sites. Subsequent membrane leakage after cytoskeleton destruction likely also contributed to enhance the bleb by increasing osmotic pressure, eventually leading to necrotic membrane rupture.

\section{Necrotic NK cell cytotoxicity is granzyme B-induced necroptosis}

NK cell-induced necrosis is poorly characterized at the molecular level. As MCF7 cells were killed mostly by this mechanism, it provided a model for probing the mechanistic origin of necrotic killing. To test the involvement of lytic granules, we used Concanamycin A (CMA), an inhibitor of vacuolar-type ATPase (VATPase) that increased the $\mathrm{pH}$ of lytic granules, to disrupt lytic granules in NK cells and inhibit their activities. As shown in Fig. 5a, b, treatment of 10 nM CMA reduced the necrotic killing of MCF7 cells from 53 to 4\% after $6 \mathrm{~h}$ of co-culture with NK cells and also abrogated the lytic granule-mediated apoptosis, which confirmed the key role of lytic granules in activating these two cytotoxic pathways. The extent of death ligand-mediated apoptosis was not affected by CMA treatment, as expected. We also used EGTA to chelate $\mathrm{Ca}^{2+}$ flux that is crucial for lytic granule transfer. We found both the necrotic death and lytic granule-mediated apoptosis in MCF7 cells were significantly decreased (Fig. 5b), again pointing to the involvement of lytic granule transfer in mediating both necrotic and granzyme B-dependent NK cell killing. Moreover, a substantial loss of necrotic MCF7 cell death was observed by treating the NK cells with a pan-granzyme inhibitor, 3,4-Dichloroisocoumarin (DCI), as well as a granzyme B-specific inhibitor, AcIEPD-CHO, illustrating that it is the granzyme activity, in particular, granzyme B activity, from the lytic granules that induces the actin depolymerization and bleb formation upon lytic granule transfer to the target cells (Fig. 5b).

Large membrane blebs dependent on the actin cytoskeleton are characteristic of necroptosis, a type of programmed necrosis that is usually induced by cell surface receptors [29-32]. This pathway has not been previously implicated in the killing by cytotoxic lymphocytes. To test its possible involvement, we knocked down key necroptosis pathway components, including receptorinteracting protein 1 (RIP1), RIP3, and mixed lineage kinase domain-like (MLKL), in MCF7 cells by RNA interference (RNAi). Loss of these three regulators all caused a significant reduction in necrotic killing and a concomitant increase in apoptotic killing (Fig. 5c). We also treated MCF7 cells with commonly used necroptosis inhibitors. The RIP3 inhibitor, GSK872, decreased necrotic MCF7 cell death to a similar degree as the RIP3 knockdown treatment (Fig. 5c). MCF7 cells are known to have very low RIP3 expression, which was attributed to their resistance to conventional necroptosis activated by death receptors [30]. We indeed observed orders of magnitude lower expression of RIP3 in MCF7 by western blot than in HT29, a cell line with high RIP3 expression. But given that both RNAi knockdown of RIP3 and the RIP3 inhibitor significantly attenuated necroptotic killing by NK cells, our results indicate the granzymeinduced necroptosis that we observed could be mediated by a low level of RIP3. We also tested the inhibitory effects of the RIP1 inhibitor, necrostatin-1 (from 10 to 100 $\mu \mathrm{M}$ ), and MLKL inhibitor, necrosulfonamide (from 1 to $10 \mu \mathrm{M})$. At low concentrations, these two inhibitors did not exhibit significant effects on attenuating necroptotic killing of NK cells, while high concentrations of both inhibitors caused substantial toxicity to the primary NK cells so we were unable to obtain definitive results regarding the inhibitory effects of these two inhibitors.

To further confirm the activation of necroptosis in MCF7 cells treated with primary NK cells, we measured the level and localization of phospho-MLKL (Ser358), a key activation signal of necroptosis, by both western blotting and immunostaining (Fig. 5d and Additional 


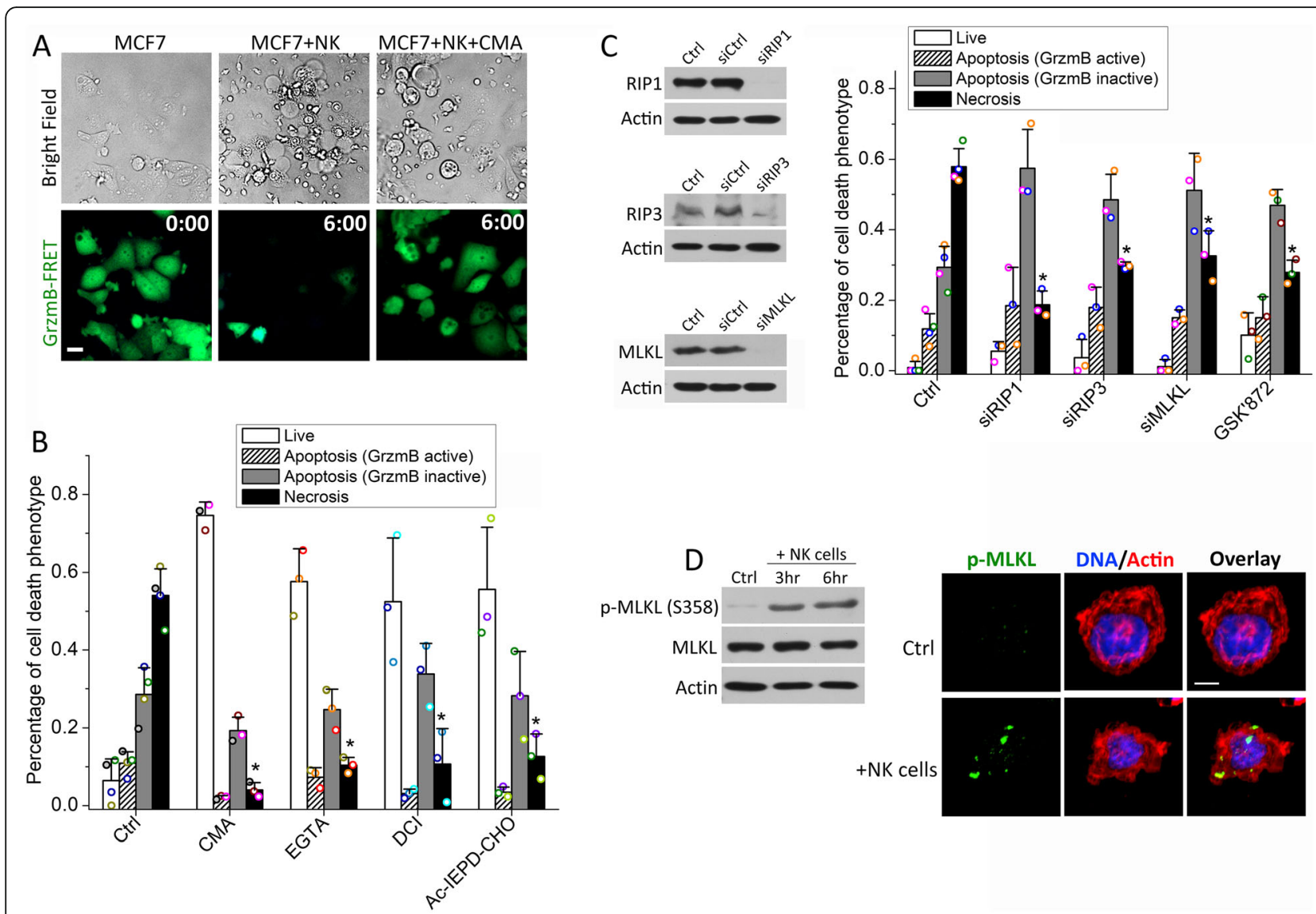

Fig. 5 Necrotic NK cell cytotoxicity is triggered by granzyme and mediated by the necroptosis pathway. a Representative phase-contrast and the corresponding GrzmB-FRET reporter images of MCF7 cells in co-culture with primary NK cells with or without 10 nM CMA (vacuolar-type ATPase inhibitor that disrupts lytic granules). Time is indicated in the unit of hours:minutes on the GrzmB-FRET reporter images. The white scale bar is 20 $\mu \mathrm{m}$. $\mathbf{b}$ Distribution of the indicated response phenotypes of MCF7 cells after 6-h co-culture with primary NK cells under the control condition or treatment of $10 \mathrm{nM}$ CMA, $0.9 \mathrm{mM}$ EGTA (calcium chelator that inhibits lytic granule transfer), $40 \mu \mathrm{M} \mathrm{DCl}$ (pan-granzyme inhibitor), and $50 \mu \mathrm{M}$ AcIEPD-CHO (granzyme B inhibitor). c Distribution of the cytotoxic response phenotypes of MCF7 cells upon knockdown of RIP1, RIP3, and MLKL, respectively, and under the treatment of $5 \mu \mathrm{M}$ GSK'872 (RIP3 inhibitor). The knockdown efficiency is demonstrated by the western blots. Data plotted in $\mathbf{b}$ and $\mathbf{c}$ were averaged from 3 independent imaging experiments, and the number of cells analyzed for each condition/experiment ranges from 55 to 112. The error bars are standard deviations. Data acquired with NK cells from the same donors are denoted with the same color symbols in each sub-figure. P values were obtained by Student's t test comparing the treatment condition with control. ${ }^{*} \mathrm{P}<0.001$. d Left panel: western blot analysis of phospho-MLKL upregulation in MCF7 cells in co-culture with primary NK cells for $3 \mathrm{~h}$ and $6 \mathrm{~h}$, respectively. Right panel: representative images of phospho-MLKL immunofluorescence signal in MCF7 cells under control vs. in co-culture with primary NK cells. The white scale bar is $5 \mu \mathrm{m}$

file 1: Figure S6). We found significant upregulation of phospho-MLKL in MCF7 cells upon 3-h co-culture with NK cells. The induction level of phospho-MLKL did not further increase significantly at $6 \mathrm{~h}$ of NK-MCF7 cell coculture, agreeing with our observation from time-lapse imaging that most necrotic NK cell killing occurred early within the first $3 \mathrm{~h}$, while apoptotic killing accounted for the majority of late MCF7 cell death after $3 \mathrm{~h}$. Moreover, immunofluorescence analysis revealed NK cell treatment induced distinctive phospho-MLKL puncta that indicated pore formation. Together with the RNAi data above, our results demonstrated the necrotic NK cell killing that we observed was granzyme B-induced necroptosis.

\section{Discussion}

Our main novel finding concerns variability in NK cell killing mode between different epithelial targets and the cellular determinants of this choice, in particular, for the previously unknown necroptotic killing. As necrotic death releases cell debris to "inflame" the local microenvironment, altering the NK cell cytotoxic modes, i.e., necroptosis vs. apoptosis, by modulating target membrane dynamics can enhance or damp the secondary inflammatory response subsequent to NK cell killing. This may hold the potential of, e.g., amplifying the anti-tumor effect of adoptive cell transfer therapy beyond primary NK cell killing. Moreover, our result that membrane dynamics determine sensitivity to necroptotic killing has 
an interesting implication for cancer, and perhaps for the role of necroptosis in homeostasis and disease more broadly. The Rho family of GTPases, e.g., Rac1, and the associated kinases, e.g., ROCK, are involved in key oncogenic signaling pathways, particularly by activating the actomyosin network and promoting cancer invasion and metastasis [33, 34]. Upregulation of Rac1 activity has been frequently observed in human cancer [35] and Rac1 inhibitor, such as NSC23766 that we used in this study, is being actively pursued as anticancer therapeutic. Our data showed that tuning membrane dynamics by targeting Rac1 and ROCK is an effective strategy to influence the cytotoxic mode of NK cells. For cancers with elevated activity of Rac1 and/or Rho/ROCK signaling, they are likely more sensitive to proinflammatory killing by NK cells via necroptosis. Alternatively, inhibitors of the RTK signaling pathways that block protrusive and contractile membrane activity could be possibly employed to promote apoptotic killing in NK cell therapy. Necroptosis has been implicated as a major tissue damage pathway in diverse diseases, including stroke and neurodegeneration [36, 37], and is activated by death receptor in combination with caspase inhibition [32]. Our finding that necroptosis can be triggered by granzyme $\mathrm{B}$ and that alteration in the actomyosin network and cell cortex may also be involved provide a novel mechanistic angle to investigate alternative regulatory pathway(s) underlying necroptosis in other disease contexts.

In terms of the mechanism underlying NK cellinduced necroptosis, our results suggested that a highly dynamic membrane, e.g., in MCF7 cells, likely facilitates excessive lytic granule transfer and subsequently results in rapid induction of high-level granzyme B activity. Such a high level of activated granzyme may preferentially cleave substrates associated with the actomyosin network, e.g., the ERM family proteins (actin-membrane linkers) [38], leading to actin depolymerization and collapse of the cell cortex. Previous studies of mechanosensing involved in $\mathrm{T}$ cell activation showed that tension generated at the plasma membrane interphase of immune-target cell conjugation indeed facilitates lytic granule release to the target cell, providing a possible mechanistic link between membrane dynamics and lytic granule transfer via force induction [39]. In addition, it was reported that accumulation of F-actin at the NKtarget cell IS attenuated lytic granule transfer and the subsequent NK cell cytotoxicity towards epithelial cancer targets [40]. Therefore, the actin depolymerization at the IS, i.e., loss of F-actin, which we observed upon necroptotic NK cell killing, may very well contribute to promote lytic granule transfer. However, it is unclear whether the necroptosis machinery is activated due to the disruption of the actomyosin network and cell cortex or is activated directly by the proteolytic substrates of granzyme B. Yet to be resolved by follow-up studies are also the important mechanistic questions of how granzyme B activates the phosphorylation of RIP1 and RIP3, leading to the induction of necroptosis.

Our measurements of the different NK cell receptor ligands for tuning overall sensitivity suggest that it should be possible, in principle to predict, and eventually modulate, the sensitivity of different cancers to NK cell therapy. However, the relationship between receptor expression and sensitivity is complex, and measurement of one or two ligands will not be sufficient. For example, we found the non-cancer cell line, LO2, which was the most resistant to NK cell cytotoxicity, expressed the highest level of NKG2D ligand, MICA, and also a high level of ULPs when compared with the cancer cell lines (Fig. 2b). Previous clinical studies have also shown that high levels of NKG2D ligands were not associated with the extent of NK cell infiltration into the tumors or better prognosis for patients with breast, lung, or ovarian cancers [41], suggesting that expressions of NK cellactivating ligands likely vary significantly between cancer types and tumor context, thus hindering their standalone prognostic potential.

\section{Conclusions}

Our study revealed that epithelial cell targets varied not only in their overall sensitivity to killing by primary NK cells, but also in the mode of killing. Overall sensitivity was determined primarily by inhibitory and activating receptor-ligand interactions, consistent with previous literature. However, these interactions did not specify the killing mode. Rather, membrane dynamics of the epithelial targets appeared to play a central role in cytotoxic mode specification. We also demonstrated necroptosis as an effector mechanism in NK cell killing for the first time. These findings have important implications for immunotherapy of solid tumors and for understanding the regulation of necroptosis in other homeostatic and disease processes.

\section{Methods}

\section{Isolation of primary NK cells from human blood}

Primary human NK cells were isolated from fresh human buffy coat of healthy donors (within $24 \mathrm{~h}$ of blood donation) obtained from the Hong Kong Red Cross. The buffy coat was first separated by Ficoll-Paque Plus solution and NK cells were then purified from the Peripheral Blood Mononuclear Cells (PBMCs) by negative selection using the EasySep Human NK Cell Enrichment Kit (STEMCELL Technologies), according to the manufacturer's protocol. Only NK cell batches with purity higher than 90\% (measured by FACS analysis of CD56 staining) were used for experiments. Fresh human NK cells were 
cultured for 3 days at a density of $1 \times 10^{6}$ cells $/ \mathrm{ml}$ in RPMI 1640 medium (Gibco, Thermo Fisher) containing $1 \mathrm{ng} / \mathrm{ml}$ recombinant human IL-2 (PeproTech), 10\% heat-inactivated Fetal Calf Serum (Gibco, Thermo Fisher), $100 \mathrm{U} / \mathrm{ml}$ penicillin, and $100 \mu \mathrm{g} / \mathrm{ml}$ streptomycin (Gibco, Thermo Fisher), prior to experiments. Experimental data were averaged from 3 independent experiments, and each experiment used NK cells purified from a different healthy donor.

\section{Cell lines and cell culture}

The live-cell Förster resonance energy transfer (FRET) construct that reports on the granzyme-B proteolytic activity as well as the mitochondrial reporter, IMS-RP, were engineered into the five selected epithelial cell lines by retroviral infection, as described previously [20, 21]. Isogenic clones of the fluorescent reporter cell lines that exhibited cell death response to primary NK cell killing most similar to their respective parental lines were selected for performing the live-cell imaging assays. Cell lines were cultured in appropriate medium supplemented with $10 \%$ fetal calf serum, $100 \mathrm{U} / \mathrm{ml}$ penicillin, and $100 \mu \mathrm{g} / \mathrm{ml}$ streptomycin. Specifically, U-2 OS was maintained in McCoy's 5A (modified) Medium; MCF7 was maintained in RPMI 1640 Medium; SMMC-7721, HeLa, and LO2 were maintained in Dulbecco's modified Eagles medium (DMEM). For all co-culture experiments with primary NK cells, the NK-to-target cell ratio of 3:1 was used and $25 \mathrm{ng} / \mathrm{ml} \mathrm{IL-2}$ was supplemented in the medium. To image the dynamics of F-actin, we transiently transfected MCF7 and SMMC-7721 cells with the Utrophin-GFP reporter (Plasmid \#26737, Addgene) using X-tremeGENE HP DNA Transfection Reagent (Roche). Time-lapse imaging was performed $48-72 \mathrm{~h}$ after the transfection.

\section{Chemicals and neutralizing antibodies}

The following inhibitors were purchased from Selleck Chem: NSC23766 (Rac1 inhibitor; used at $120 \mu \mathrm{M}$ for MCF7 and $50 \mu \mathrm{M}$ for SMMC-7721), necrostatin-1 (RIP1 inhibitor; titrated from 10 to $100 \mu \mathrm{M}$ ), GSK'872 (RIP3 inhibitor; used at $5 \mu \mathrm{M}$ ), and necrosulfonamide (MLKL inhibitor; titrated from 1 to $10 \mu \mathrm{M}$ ). Y27632 (ROCK inhibitor; used at $5 \mu \mathrm{M}$ ), Concanamycin A (Vacuolar type ATPase inhibitor; used at $10 \mathrm{nM}$ ), 3,4-Dichloroisocoumarin (pan-granzyme inhibitor; used at $40 \mu \mathrm{M}$ ), and AcIEPD-CHO (granzyme-B inhibitor; used at $50 \mu \mathrm{M}$ ) were purchased from Sigma, Santa Cruz Biotechnology, Cayman Chemical, and Biovision, respectively. Cells were pre-treated with the inhibitors for $2 \mathrm{~h}$ before experiments, except for NSC23766 (5-12 h pre-treatment), Concanamycin A (4-h pre-treatment), and 3,4-Dichloroisocoumarin (20-min treatment and then the inhibitor was removed to prevent toxicity to NK cells in the co- culture experiments). To block the activity of various NK cell receptors, the following neutralizing antibodies from Biolegend were used: Purified anti-human HLA-A, B,C antibody (Clone W6/32), CD94 (Clone DX22), NKG2D (Clone 1D11), NKp46 (Clone 9E2), DNAM-1 (Clone 11A8), and CD16 (Clone 3G8). Control NK cells were blocked with the mouse IgG1 $\mathrm{k}$ Isotype Control (Clone MOPC-21) to control for non-specific effects. Except for the HLA-A,B,C and CD16 antibody, which were used at $5 \mu \mathrm{g} / \mathrm{ml}$, the rest of the antibodies were used at $10 \mu \mathrm{g} / \mathrm{ml}$. In the blocking experiments, either the NK cells or the target cells were pre-treated with the neutralizing antibody or combination of antibodies for $1 \mathrm{~h}$ before the co-culture analysis.

\section{Gene knockdown by RNAi}

siRNA oligos for knocking down MICB (5'-CATTCCAT TGGAGGCTATATGATCA-3'), RIP1 (5'-CCACUA GUCUGACGGAUAA-3'), RIP3 (5'-CCAGAGACCU CAACUUUCA-3'), and MLKL (5'-CAAACUUCCU GGUAACUCA-3') were custom synthesized by Dharmacon. Dharmacon On-Target plus siControl (\#D001810-01, 5'-UGGUUUACAUGUCGACUAA-3') was used as non-targeting siRNA control. MICB siRNA oligo was used at $40 \mathrm{nM}$, RIP1 siRNA at $40 \mathrm{nM}$, RIP3 siRNA at $60 \mathrm{nM}$, and MLKL at $30 \mathrm{nM}$. siRNA transfections were performed using Lipofectamine (Thermo Fisher) according to the manufacturers' instructions. Experiments were conducted after $48 \mathrm{~h}$ of gene silencing. Gene knockdown efficiency was probed by western blot using the following primary antibodies: RIP1 (\#3493), RIP3 (\#13526), and MLKL (\#14993) from Cell Signaling and MICB (\#MAB1599) from R\&D Systems. Western blotting was performed using standard protocol, e.g., as described in [23].

\section{Time-lapse microscopy and image analysis}

Cells were plated in 96-well $\mu$-plate (ibidi) and cultured in $\mathrm{CO}_{2}$-independent medium (Gibco, Thermo Fisher) supplemented with $10 \%$ heat-inactivated FCS, $4 \mathrm{mM}$ Lglutamine, $100 \mathrm{U} / \mathrm{ml}$ penicillin, and $100 \mu \mathrm{g} / \mathrm{ml}$ streptomycin. Cells were imaged either by a Nikon Eclipse TE2000 inverted microscope enclosed in a humidified chamber maintained at $37^{\circ} \mathrm{C}$ with a $\times 20$ plan Apo objective $(\mathrm{NA}=0.95)$, Xcite XYLIS LED fluorescence illumination, and Zyla 4.2 Plus camera; or by the Andor Dragonfly confocal microscope with a $\times 40$ objective $(\mathrm{NA}=0.95)$, laser illumination and Zyla 4.2 Plus camera fitted with an incubation chamber. Images were acquired every $30 \mathrm{~s}$ to $4 \mathrm{~min}$ with the following filter sets and exposure time: (1) GzmB-FRET (CFP FRET, 35-90 ms; YFP FRET, 35-90 ms), (2) IMS-RP (TxRed, 80-150 ms), (3) LysoBrite (TxRed, 60-100 ms), and (4) UtrophinGFP (GFP, 100-200 ms). 
Data were viewed and analyzed using the NIS software (Nikon) or Imaris. Target cell death was scored morphologically by blebbing followed by cell lysis, and the time from NK cell addition (i.e., time 0) to morphological target cell death was plotted as cumulative survival curves, in which the number of surviving live target cells was ratioed to the total number of live target cells at time 0 as a function of time. The onset of apoptosis is scored by an abrupt transition from punctate to smooth localization of the mitochondria reporter, IMS-RP. To quantify the time course of the GrzmB-FRET reporter signal, ImageJ was used to segment the individual cells and measure the total intensity of YFP and CFP signal, respectively.

\section{Flow cytometry analysis}

$1 \times 10^{5}$ epithelial target cells were washed and resuspended in the cell staining buffer (Biolegend) containing different dye-conjugated primary antibodies at 1 $\mu \mathrm{g} / \mathrm{ml}$ or $5 \mu \mathrm{g} / \mathrm{ml}$ for $30 \mathrm{~min}$ at $4^{\circ} \mathrm{C}$. Cells were then washed and stained with the Zombie NIR (cell death marker, Biolegend). The stained cells were analyzed by flow cytometry with a FACSCanto II cytometer (BD Biosciences). Primary antibodies targeting the various surface ligands and receptors as well as the corresponding control include: FITC anti-human HLA-A,B,C (Clone W6/32, Biolegend), PE anti-human HLA-E (Clone 3D12, Biolegend), FITC anti-human ICAM-1/CD54 (Clone DX2, Biolegend), FITC anti-human Fas/CD95 (Clone DX2, Biolegend), APC anti-human Nectin-2/CD112 (Clone TX31, Biolegend), APC anti-human PVR/CD155 (Clone SKII.4, Biolegend), PE anti-human MICA (Clone 159227, R\&D Systems), PE anti-human MICB (Clone 236511, R\&D Systems), PE anti-human ULBP-2/5/6 (Clone 165903, R\&D Systems), FITC Mouse IgG1 к Isotype Control (Clone MOPC-21, Biolegend), APC Mouse IgG1 к Isotype Control (Clone MOPC-21, Biolegend), and PE Mouse IgG2b к Isotype Control (Clone MPC-11, Biolegend). Cell death analysis by Annexin V staining was performed using the FITC Annexin V detection kit (Biolegned) following the manufacturer's protocol.

\section{Immunofluorescence analysis}

MCF7 cells grown on glass coverslips and in co-culture with primary NK cells for $3 \mathrm{~h}$ were fixed in 3.7\% paraformaldehyde (PFA)/phosphate-buffered saline (PBS) for 15 min, permeabilized with $0.5 \%$ Triton-X100 for $30 \mathrm{~min}$, washed and blocked in 5\% BSA/0.1\% Triton-X100/PBS for $1 \mathrm{~h}$, and then incubated with the primary antibody against phospho-MLKL (S358) (\#ab187091, Abcam) and the fluorescently conjugated Alexa-Fluor secondary antibody (Invitrogen), as well as stained with Hoechst (Invitrogen) and Phalloidin (Alexa Fluor 647, Invitrogen). All procedures were conducted at room temperature, except for phospho-MLKL primary antibody binding (overnight at $4{ }^{\circ} \mathrm{C}$ ). The coverslips were mounted on glass slides with ProLong Gold antifade reagent (Invitrogen) and imaged using the Andor Dragonfly spinning disc confocal microscope with a $60 x$ objective.

\section{Supplementary Information}

The online version contains supplementary material available at https://doi. org/10.1186/s12915-021-01068-3.

Additional file 1: Figure S1. FACS analysis of NK cell-induced target cell death. Figure S2- Effect of MICB knockdown on NK cell killing. Figure S3. Cancer target-dependent kinetics and phenotypes of NK cell killing. Figure S4. NK cell killing under NK CD16 neutralization. Figure S5. Membrane distributions of HLA-A,B,C in MCF7 cells and KIR2D in primary NK cells by immunostaining. Figure S6. Additional representative immunofluorescence images of phospho-MLKL. Figure S7. Full western blots.

Additional file 2: Movie S1. Time-lapse movie of MCF7 cell expressing the GrzmB-FRET reporter (green and blue) in co-culture with primary NK cells stained by Lysobrite (red). The image is the overlay of the GzmB FRET reporter, Lysobrite and bright-field signals. Time is indicated in the unit of hour:minute.

Additional file 3: Movie S2. Time-lapse movie of SMMC-7721. The image is the overlay of the GzmB FRET reporter, Lysobrite and bright-field signals.

Additional file 4: Movie S3. Confocal live-cell imaging of F-actin (green) in MCF7 cell in co-culture with NK cells stained with Lysobrite (red).

Additional file 5: Movie S4. Confocal live-cell imaging of F-actin (green) in SMMC-7721 cell in co-culture with NK cells stained with Lysobrite (red).

\section{Acknowledgements}

We thank Dr. Paul Choi and Dr. Timothy Mitchison (Department of Systems Biology, Harvard Medical School) for the granzyme-B FRET reporter construct and Dr. John Albeck and Dr. Peter Sorger (Department of Systems Biology, Harvard Medical School) for the IMS-RP retroviral vector.

\section{Authors' contributions}

J.S. designed and supervised the study. Y.Z. performed the experiments. Y.Z., J.X., and J.S. analyzed the data. Y.Z. and J.S. wrote the paper. All authors read and approved the final manuscript.

\section{Funding}

This work was supported by the Hong Kong Research Grant Council (\#12101514, \#T12-710/16-R and \#C2006-17E) to J. Shi and the "Laboratory for Synthetic Chemistry and Chemical Biology" under the Health@InnoHK Program by the Innovation and Technology Commission of Hong Kong.

Availability of data and materials

All data generated or analyzed during this study are included in this article. Additional representative images of NK-cancer cell interaction as shown in Fig. 4 are deposited and available at figshare [42].

\section{Declarations}

Ethics approval and consent to participate

Blood samples of healthy donors were obtained from the Hong Kong Red Cross Transfusion Center. We had no contact with or information about the individual donors. All the ethics approval and consent forms were handled by the Hong Kong Red Cross under their own ethics guidelines. The blood samples were not collected solely for our work, and we were using existing blood samples made available to the public for medical research purposes.

Consent for publication

Not applicable. 


\section{Competing interests}

The authors declare that they have no competing interests.

Received: 21 April 2021 Accepted: 9 June 2021

Published online: 30 July 2021

\section{References}

1. Caligiuri MA. Human natural killer cells. Blood. 2008;112(3):461-9. https://doi. org/10.1182/blood-2007-09-077438.

2. Freud AG, Mundy-Bosse BL, Yu J, Caligiuri MA. The broad spectrum of human natural killer cell diversity. Immunity. 2017:47(5):820-33. https://doi. org/10.1016/j.immuni.2017.10.008.

3. Chiossone L, Dumas PY, Vienne M, Vivier E. Natural killer cells and other innate lymphoid cells in cancer. Nat Rev Immunol. 2018;18(11):671-88. https://doi.org/10.1038/s41577-018-0061-z

4. Ruscetti M, Leibold J, Bott MJ, Fennell M, Kulick A, Salgado NR, et al. NK cellmediated cytotoxicity contributes to tumor control by a cytostatic drug combination. Science. 2018;362(6421):1416-22. https://doi.org/10.1126/ science.aas 9090

5. Souza-Fonseca-Guimaraes F, Cursons J, Huntington ND. The Emergence of Natural Killer Cells as a Major Target in Cancer Immunotherapy. Trends Immunol. 2019:40(2):142-58.

6. Lanier LL. NK cell recognition. Annu Rev Immunol. 2005;23(1):225-74. https://doi.org/10.1146/annurev.immunol.23.021704.115526.

7. Bryceson YT, March ME, Ljunggren HG, Long EO. Activation, coactivation, and costimulation of resting human natural killer cells. Immunol Rev. 2006; 214(1):73-91. https://doi.org/10.1111/j.1600-065X.2006.00457.x.

8. Smyth MJ, Swann J, Cretney E, Zerafa N, Yokoyama WM, Hayakawa Y. NKG2D function protects the host from tumor initiation. J Exp Med. 2005; 202(5):583-8. https://doi.org/10.1084/jem.20050994.

9. Champsaur M, Lanier LL. Effect of NKG2D ligand expression on host immune responses. Immunol Rev. 2010;235(1):267-85. https://doi.org/1 0.1111/j.0105-2896.2010.00893.x.

10. Cho D, Shook DR, Shimasaki N, Chang YH, Fujisaki H, Campana D. Cytotoxicity of activated natural killer cells against pediatric solid tumors. Clin Cancer Res. 2010;16(15):3901-9. https://doi.org/10.1158/1078-0432.CCR-10-0735.

11. Prager I, Watzl C. Mechanisms of natural killer cell-mediated cellular cytotoxicity. J Leukoc Biol. 2019;105(6):1319-29. https://doi.org/10.1002/JLB. MR0718-269R.

12. Smyth MJ, Cretney E, Kelly JM, Westwood JA, Street SE, Yagita H, et al. Activation of NK cell cytotoxicity. Mol Immunol. 2005;42(4):501-10. https:// doi.org/10.1016/j.molimm.2004.07.034

13. Zhou Z, He H, Wang K, Shi X, Wang Y, Su Y, et al. Granzyme A from cytotoxic lymphocytes cleaves GSDMB to trigger pyroptosis in target cells. Science. 2020;368:eaaz7548.

14. Zhang Z, Zhang Y, Xia S, Kong Q, Li S, Liu X, et al. Gasdermin E suppresses tumour growth by activating anti-tumour immunity. Nature. 2020;579(7799): 415-20. https://doi.org/10.1038/s41586-020-2071-9.

15. Backes CS, Friedmann KS, Mang S, Knörck A, Hoth M, Kummerow C. Natural killer cells induce distinct modes of cancer cell death: discrimination, quantification, and modulation of apoptosis, necrosis, and mixed forms. J Biol Chem. 2018;293(42):16348-63. https://doi.org/10.1074/jbc.RA118.004549.

16. Voskoboinik I, Smyth MJ, Trapani JA. Perforin-mediated target-cell death and immune homeostasis. Nat Rev Immunol. 2006;6(12):940-52. https://doi.org/1 $0.1038 /$ nri1983.

17. Bradley M, Zeytun A, Rafi-Janajreh A, Nagarkatti PS, Nagarkatti M. Role of spontaneous and interleukin-2-induced natural killer cell activity in the cytotoxicity and rejection of Fas+ and Fas- tumor cells. Blood. 1998;92(11): 4248-55. https://doi.org/10.1182/blood.V92.11.4248.

18. Screpanti V, Wallin RP, Ljunggren $H G$, Grandien A. A central role for death receptor-mediated apoptosis in the rejection of tumors by NK cells. J Immunol. 2001;167(4):2068-73. https://doi.org/10.4049/jimmunol.167.4.2068.

19. Gardiner CM, Reen DJ. Differential cytokine regulation of natural killer cellmediated necrotic and apoptotic cytotoxicity. Immunology. 1998;93(4):5117. https://doi.org/10.1046/j.1365-2567.1998.00464.x.

20. Choi PJ, Mitchison TJ. Imaging burst kinetics and spatial coordination during serial killing by single natural killer cells. Proc Natl Acad Sci U S A. 2013;110(16):6488-93. https://doi.org/10.1073/pnas.1221312110.

21. Albeck JG, Burke JM, Aldridge BB, Zhang M, Lauffenburger DA, Sorger PK. Quantitative analysis of pathways controlling extrinsic apoptosis in single cells. Mol Cell. 2008;30(1):11-25. https://doi.org/10.1016/j.molcel.2008.02.012.
22. Zhu Y, Huang B, Shi J. Fas ligand and lytic granule differentially control cytotoxic dynamics of natural killer cell against cancer target. Oncotarget. 2016;7(30):47163-72. https://doi.org/10.18632/oncotarget.9980.

23. Zhu Y, Zhou Y, Shi J. Post-slippage multinucleation renders cytotoxic variation in anti-mitotic drugs that target the microtubules or mitotic spindle. Cell Cycle. 2014;13(11):1756-64. https://doi.org/10.4161/cc.28672.

24. Chan CJ, Smyth MJ, Martinet L. Molecular mechanisms of natural killer cell activation in response to cellular stress. Cell Death Differ. 2014;21(1):5-14. https://doi.org/10.1038/cdd.2013.26.

25. Bergert M, Chandradoss SD, Desai RA, Paluch E. Cell mechanics control rapid transitions between blebs and lamellipodia during migration. Proc Natl Acad Sci U S A. 2012;109(36):14434-9. https://doi.org/10.1073/pnas.12 07968109.

26. Akbar H, Cancelas J, Williams DA, Zheng J, Zheng Y. Rational design and applications of a Rac GTPase-specific small molecule inhibitor. Methods Enzymol. 2006;406:554-65. https://doi.org/10.1016/S0076-6879(06)06043-5.

27. Morelli A, Chiozzi P, Chiesa A, Ferrari D, Sanz JM, Falzoni S, et al. Extracellular ATP causes ROCK I-dependent bleb formation in P2X7-transfected HEK293 cells. Mol Biol Cell. 2003;14(7):2655-64. https://doi.org/10.1091/mbc.02-040061.

28. Burkel BM, von Dassow G, Bement WM. Versatile fluorescent probes for actin filaments based on the actin-binding domain of utrophin. Cell Motil Cytoskeleton. 2007;64(11):822-32. https://doi.org/10.1002/cm.20226.

29. Degterev A, Huang Z, Boyce M, Li Y, Jagtap P, Mizushima N, et al. Chemical inhibitor of nonapoptotic cell death with therapeutic potential for ischemic brain injury. Nat Chem Biol. 2005;1(2):112-9. https://doi.org/10.1038/ nchembio711.

30. He S, Wang L, Miao L, Wang T, Du F, Zhao L, et al. Receptor interacting protein kinase-3 determines cellular necrotic response to TNF-alpha. Cell. 2009;137(6):1100-11. https://doi.org/10.1016/j.cell.2009.05.021.

31. Sun L, Wang H, Wang Z, He S, Chen S, Liao D, et al. Mixed lineage kinase domain-like protein mediates necrosis signaling downstream of RIP3 kinase. Cell. 2012;148(1-2):213-27. https://doi.org/10.1016/j.cell.2011.11.031.

32. Weinlich R, Oberst A, Beere HM, Green DR. Necroptosis in development, inflammation and disease. Nat Rev Mol Cell Biol. 2017;18(2):127-36. https:// doi.org/10.1038/nrm.2016.149.

33. Kazanietz MG, Caloca MJ. The Rac GTPase in cancer: from old concepts to new paradigms. Cancer Res. 2017;77(20):5445-51. https://doi.org/10.1158/ 0008-5472.CAN-17-1456.

34. Cardama GA, Gonzalez N, Maggio J, Menna PL, Gomez DE. Rho GTPases as therapeutic targets in cancer (review). Int J Oncol. 2017;51(4):1025-34. https://doi.org/10.3892/ijo.2017.4093.

35. Olson MF. Rho GTPases, their post-translational modifications, diseaseassociated mutations and pharmacological inhibitors. Small GTPases. 2018; 9(3):203-15. https://doi.org/10.1080/21541248.2016.1218407.

36. Choi ME, Price DR, Ryter SW, Choi AMK. Necroptosis: a crucial pathogenic mediator of human disease. JCI Insight. 2019;4(15):e128834. https://doi.org/1 $0.1172 /$ jci.insight.128834

37. Shan B, Pan H, Najafov A, Yuan J. Necroptosis in development and diseases. Genes Dev. 2018:32(5-6):327-40. https://doi.org/10.1101/gad.312561.118.

38. Bovenschen N, de Koning PJ, Quadir R, Broekhuizen R, Damen JM, Froelich $\mathrm{CJ}$, et al. NK cell protease granzyme M targets alpha-tubulin and disorganizes the microtubule network. J Immunol. 2008;180(12):8184-91. https://doi.org/10.4049/jimmunol.180.12.8184.

39. Rossy J, Laufer JM, Legler DF. Role of mechanotransduction and tension in cell function. Front Immunol. 2018;9:2638. https://doi.org/10.3389/fimmu.201 8.02638.

40. Al Absi A, Wurzer H, Guerin C, Hoffmann C, Moreau F, Mao X, et al. Actin cytoskeleton remodeling drives breast cancer cell escape from natural killermediated cytotoxicity. Cancer Res. 2018;78(19):5631-43. https://doi.org/10.11 58/0008-5472.CAN-18-0441.

41. Sheppard S, Ferry A, Guedes J, Guerra N. The paradoxical role of NKG2D in cancer immunity. Front Immunol. 2018;9:1808. https://doi.org/10.3389/ fimmu.2018.01808.

42. Zhu Y, Xie J, Shi J. Rac1/ROCK-driven membrane dynamics promote natural killer cell cytotoxicity via granzyme-induced necroptosis. figshare doi:https:// doi.org/10.6084/m9.figshare.14685066.v1 (2021)

\section{Publisher's Note}

Springer Nature remains neutral with regard to jurisdictional claims in published maps and institutional affiliations. 